power of diagnosis. And this leads me to some observations which, I think, are most important from the clinical standpoint.

Early Signs Should be Recognised.

In order to obtain a criterion by which they could determine success or otherwise in producing rickets the workers in the experiments have been obliged to look to the changes in the bones, especially in the long bones of the limbs and in the costo-chondral junctions. It is unfortunate that no earlier acceptable criterion of the existence of the disease was available, for all clinicians will agree that the changes in the long bones and ribs are by no means early changes in rickets. When they occur they imply a period of action of the underlying cause of the disease of some considerable duration; the greater our success in recognising the disease before the period of the development of these bony changes, the more amenable to treatment is the disease. It is true that the onset of the disorder is often so insidious that frequently children are not brought to the notice of the doctor till these bony changes are well advanced, but it is also true that the doctor should not only himself be able to recognise the disease at an earlier stage, but should actively teach that the ibone changes can be prevented by attention to signs and symptoms fairly easy to determine. The prevalence of the bony deformities of rickets ought to be regarded as evidence that the doctor has hitherto failed in his duty to the community. So much attention has been in the past paid to the surgical correction of bone deformities that as a profession we have failed to enforce the doctrine that such changes ought never to be allowed to occur; no child should reach the stage where bony deformities require direct interference.

The early signs on which I should lay stress are five in number: (1) Head-sweating and restless sleep; (2) loss of muscle-tone; (3) polyuria; (4) pallor; (5) alteration in the bones of the skull.

The normal infant of six months or less sleeps without more than a moisture of the skin, wakes for its food, and then sleeps peaceably again. If in sleep beads of perspiration appear about the face and neck, unless there is some obvious illness the baby has rickets.

Even moderate sweating accompanied by undue restlessness and wakefulness has rickets for its explanation; but sometimes the sweating is replaced by loss of fluid from the urinary tract. The mother will tell you that it is quite impossible to keep the baby dry. Such polyuria is a frequent sign of rickets, and even were it unaccompanied by any other sign would arouse suspicion. But there is another sign almost invariably present. The normal baby has firm muscles; when awake he is in constant movement; and the least touch convinces the observer that the tone of the muscles is excellent. The rickety child, on the contrary, is slow to more, takes no delight in exercising his muscles, and these are found slack, soft. and slow to respond by contracting. This loss of muscle-tone is an early and characteristic sign of rickets. Pallor is not invariable, but is a common accompaniment of rickets. Yet some rickety children retain a good colour and any serious degree of anæmia is not a sign of rickets alone. It may be an indication of a slight degree of scurvy. The earliest changes in the bones are found in the bones of the skull. Often before there is any perceptible change in the long bones there is a softening in the lines of the sutures of the skull and the anterior fontanelle remains unduly wide. With this there is a delay in the eruption of the teeth and often a malformation of these when they do appear.

When any of these five symptoms are present there should be a suspicion of rickets and the diet and hygiene of the infant should be investigated and the known factors of rickets eliminated. If this is consistently done the fear of bony deformities is dissipated, for there is no disorder which yields so promptly to the appropriate treatment as early rickets.
When we attempt to answer the question whether the cause of rickets is known we must answer that the experimental work has up till now failed to provide a satisfactory theory. "The " vitamin " hypothesis is so far not proved, and there are, as I have attempted to show, some cogent reasons for believing that rickets is not essentially a "deficiency" disease. Yet all clinical experience tends to support the idea that the diet has a large share in its production, and that the absence or deficiency of animal fats is an important factor. So far clinical experience coincides with the experimental work of Mellanby and others, but clinical experience also has long recognised that the factors on which the opposing school lays stress, the general unhygienic conditions associated with insanitary, illventilated, and ill-lighted houses, are almost equally important. As clinicians we cannot give our undivided support to either hypothesis, and must recognise that the work of both schools has tended to confirm and establish the traditional views which were so clearly set forth by Cheadle at this hospital a quarter of a century ago.

\section{THE PELVIC APPENDIX.}

\section{By RUTHERFORD MORISON, M.D. EDIN.,} F.R.C.S. ENG.,

EMERITUS PROFESSOR OF SURGERY, UNIYERATTY OF DURHAM.

So much has been written and said and done about appendicitis during the last 20 years that there may well seem nothing left to be told, but so long as the pelvic appendix still frequently betrays experienced and skilful surgeons, it is an obvious duty to contribute towards the lessening of the difficulties connected with it. In a paper published in the Edinburgh Medical Journal 28 vears ago ${ }^{1}$ I made this statement: "It is probakly correct to say that sudden severe abdominal pain attended by a rise in temperature means mischief in the appendix." The aphorism, based upon recognition of the importance of general surgical principles, has stood me in such good stead that I should like to emphasise it. The general signs of inflammation are comprised in the term "fever" ; of the local signs, the redness, the swelling, and the heat are invisible and inappreciable before the appendix is exposed, but in the ordinary case the right iliac protective muscular rigidity and local tenderness are additional invaluable aids to diagnosis. Remembrance of the aphorism has been most useful to me in pelvic cases, for without this a diagnosis of appendicitis would never have suggested itself in many of them. Fven moderate requirements like this may not be satisfied, and an early diagnosis, which is so important in these cases, may be missed if too much reliance be placed upon such hints or ordinary signs. Much of the material I am using to illustrate points raised is taken from my old case notes, republished from papers in many different medical journals in "Surgical Contributions," Vol. II., and the references to pages throughout this paper apply to this book of mine.

\section{Diagnosis.}

Why is the diagnosis in pelvic appendix cases difficult? Over and over again it has been explained to me on seeing such cases that the patient had so little pain and seemed to be so well that no dangerous development was thought of. The absence of serious pain or discomfort was always emphasised, because this has been one of the chief diagnostic symptoms to be rolied upon in ordinary cases. Like cancer, appendicitis has popularly come to be associated with severe pain, and in both serious delay may occur as a consequence of its absence. The pain of appendicitis before rupture or gangrene of the appendix has occurred is, like nearly all surgical pain, due to tension; if there is drainage from the cavity of the inflamed appendix, pain is never severe; if, on the 
other hand, the appendix cannot empty itself, pain, for the first few hours of a serious colicky character, later becoming continuous, is an urgent symptom. $\Lambda$ stercorolith or an old stricture may be the cause of the block, but more commonly neither of these is found. In 1900 (p. 347) I offered an explanation of total gangrene of the appendix after careful examination of many specimens of normal intestines, and it was :- -

"The appearance of the appendix in these severe types is very characteristic; it looks like a large tense yellow sausage. On section it is found to contain a few drops to as many drachms of stinking fluid and the walls are completely necrotic. . . . Just at the cæeal attachment of the appendix there is often a thickened band or collar of peritoneum. When the mucous membrane about this point becomes inflamed the walls very soon become apposed and the cavity of the appendix is converted into a closed sac. The exudation from the mucous membrane continues, the organ becomes more and more distended, and the increasing pressure of the contents, unless relieved by discharge into the cæcum or by perforation, produces total gangrene."

In describing the symptoms produced by this type of appendicitis, I say (p. 813) :-

The symptoms produced are similar to those of a strangulated hernia, because they depend upon the same causes, viz., acute distension and vascular disturbance in a piece of bowel. There are acute pain, urgent vomiting, and inability to pass flatus. But there is no hernia, and there is a rise in temperature and marked tenderness and rigidity in the right iliac fossa.

If this type of appendix hangs into the pelvis and becomes inflamed there should be no real difficulty in diagnosis in the early stages, because there are urgent symptoms before gangrene or, perforation has occurred. The usual long pelvic appendix is not, I believe, of this type so often as the ordinarily placed organ is. It has retained some of its fotal characteristics and has a wide funnel-shaped opening into the cæcum, which is difficult to block by inflammatory swelling. With this type of appendix urgent symptoms are not caused by inflammation, unless a stercorolith or an old stricture happen to be present. Drainage of the inflammatory exudates consequently occurs, and until the surrounding peritoneum is seriously infected the patient makes no extraordinary complaint. It is in this type of appendix that a " natural cure" so frequently follows inflammation (see for illustration Vol. I. "Surgical Contributions," p. 312).

\section{Illustrative Cases.}

Other points in diagnosis, prognosis, and treatment will be emphasised by the relation of cases.

CAsE 1.-The child, aged 1 year and 11 months, of a capable and experienced medical man was first brought to a private hospital under my care on Jan. 27th, 1914, at 9.30 A.M. Her illness began on Jan. 23rd, when her nurse, thinking she had abdominal pain, asked her father to see her. He suspected appendicitis, and in the evening felt confident of this, as her temperature was $103 \cdot 4^{\circ} \mathrm{F}$., she had vomited twice, and was tender and rigid in the right iliac fossa. Next day two distinguished surgeons made a diagnosis of tuberculous cæcum, and two equally distinguished physicians thought the condition was appendicitis. When $I$ saw her on the fourth day of her illness there was a large tender right iliac swelling, and a temperature of $105^{\circ}$. An hour after her admission I operated and a large quantity of pus under considerable pressure was discharged. Two drainage-tubes were introduced, one at each end of the wound, which was closed by sutures between them. Owing to the serious condition of the baby no search was made for the appendix. She was sent home entirely recovered in 12 days. From that time until September, 1921, she was well, then she became pale and anæmic, lost her appetite, and was occasionally sick. A physician who was consulted thought she might be developing chorea, but after three or four weeks' treatment she much improved.

On Oct. 31st, 1921, she had some pain in the abdomen. Her father found some tenderness and a little rigidity in the muscles overlying the right iliac fossa. I saw her with him the same afternoon-nearly seven years after her operation. She was a very sensitive and nervous, but healthy-looking, quite cheerful little girl, who moved about briskly and freely, had normal pulse and temperature, ate a large raw apple with evident satisfaction, and walked to the private hospital-about a quarter of a mile-without help. She was taken in for observation, and examination of her blood showed a lencocytosis of 30,000 . The same evening her temperature was normal, but she slept fitfully all night and had occasional pain, and in the morning her temperature was $101^{\circ}$ Operation was performed at mid-day of Nov. 1st, 1921. The old scar was excised and the abdomen opened by an oblique incision in the right iliac fossa. The small intestine exposed was redilened, and some purulent fluid escaped. The appendix was found dipping over the pelvic brim, hanging into the pelvis and firmly adherent to the bottom of the pouch of Douglas. Fully $\frac{1}{2}$ oz. of pus escaped from the pelvis. The excised appendix was acutely inflamed throughout and perforated at its tip. A single drainage-tube was used, and the patient went home healed and well on the $26 \mathrm{th}, 25$ days after the operation.

CASE 2 (p. 200).—" $A$ very big, stout man, aged 42 . He had never been ill before, but two days previous to the sudden and severe commencement of his present illness, he had felt some uneasiness in his bowels. There was no bad pain, and he went about his duties and was able to sleep. Early on the morning of the third day he was awakened by an agonising pain all over the bowels, which made him feel sick and faint and cold and sweating. On July 23rd, 1895, eight days after the illness commenced, an oblique incision was made over the cæcum which was exposed. I could feel the firm, enlarged appendix dipping over the pelvic brim and some foetid pus escaped. A drainage-tube was left in the pelvis. Post-mortem showed a gangrenous perforation of

CASE 3 (p. 383).-." A man, aged 41, was admitted to a private hospital on June 20th, 1900, after an indefinite abdominal illness of one week's duration. In the right iliac region there was a distinctly tender point, but no rigidity or swelling could be felt. Bimanually (a finger of the right hand in the rectum and the left hand on the front of the abdomen) a definite tender tumour of the size of a large orange could be felt hanging into the pelvis and fixed to the pelvic brim." He recovered after removal of the appendix and drainage of the abscess.

\section{Remarks.}

Ordinary rules for the diagnosis of appendicitis are inapplicable to cases of this type. Abdominal uneasiness rather than pain, an absence of right iliac rigidity or tenderness, no vomiting, no threatening symptoms, make it easy to come to the conclusion that there is nothing seriously wrong. All the same, these cases are as dangerous as any, and more so than most, unless an early operation for the removal of the organ is done. Occasionally, especially in boys, frequency of micturition occurs as an early symptom, and I have seen such cases lead skilful doctors astray, because, especially if Bacillus coli had been discovered in the urine, the attack was attributed to bladder inflammation. In rare cases $I$ have known retention of urine the first warning of danger, but in these instances a pelvic abscess was always found on rectal examination.

\section{Rectal Examination.}

The perfunctory performance of rectal examination is of little value -it must be carefully carried out. In the earliest stages of the illness tenderness on the right side as compared with the left of the pelvis is suggestive. If the appendix can be felt, as it sometimes can, the diagnosis is nearly certain. It is not quite so, as other conditions-e.g., a stone in the lower part of the ureter-have misled me. Every tube in the body resents the intrusion of an unaccustomed stimulus on the first occasion, and the rectum is no exception. A first rectal examination produces disagreeable sensations, which in sensitive subjects may amount to pain, and except when only gross conditions are present it has to be made with skil and care to discover anything. This is the first point I want to make with regard to these difficult cases: A careful rectal examination should be made on any patient with an acute abdominal illness attended by uneasiness or pain and in which a definite diagnosis cannot be reached.

A second point is the importance of a blood count. It has surprised me to know how often this is still neglected even in hospital practice in this country. A leucocytosis of anything over 10,000 chiefly polymorphs is definite evidence of an infection somewhere in the body. In a large number of instances this has helped me as the deciding factor in favour of operation in pelvic and other dangerous but obscure cases of appendicitis. If a diagnosis has not been made till 
perforation of the pelvic appendix has occurred, the rule then is that a sudden pain and general disturbance sufficiently alarming to stir up everyone concerned follows, and if the perforated appendix is not immediately removed a pelvic abscess more or less quickly develops.

\section{Treatment.}

So early as 1894 (p. 109) and again in 1896 (p. 222) $I$ emphasised as strongly as possible my objections to the use of purgatives which then had strong advocacy and support.

" On the question as to whether purgatives or enemata should be administered in the early stages (of appendicitis), I hold strong views. That benefit may follow their use in certain cases I do not care to deny, but that a bad attack may be prevented or removed by a purgative or an enema my pathological information will not allow me to believe. The cases that get well after a purgative or an enema are those which would have got well, possibly more slowly, without either; whereas a purgative or an enema has so frequently been the precursor of urgent symptoms in cases $I$ have seen that there is no doubt left in my mind that the aggravation of symptoms was the result of this form of treatment."

Since then surgeons have over and over again repeated this warning, but even now I seldom see a patient to whom castor oil has not been given. Twenty-eight years seems a long time to take to learn and digest a surgical lesson such as this, and it is now time that it was assimilated. Abdominal distress now demands thorough careful questioning, observation, and examination with the object of making a diagnosis as to the cause of the disturbance possible before doing anything else.

\section{Pelvic Abscess.}

A pelvic abscess, the result of appendicitis, though the usual consequence of a pelvic appendix, not infrequently follows a less direct infection. Indeed, extension from a right iliac appendix is the most common cause of pelvic abscess. This may renuain a local condition or spread by continuity over the pelvic brim reaching the left iliac fossa; or it may be the result of an infection which has spread by contiguity from the appendix into the general peritoneal cavity causing at first diffuse infection, later becoming localised in the pelvis and perhaps elsewhere. The first type is so well known as to require no description here. The following case is a good example of the second type, which is fortunately a less common finding since early operation became the rule.

CASE 4 (p. 522).- "Male, aged 15, admitted to the infirmary on Sunday, August 3rd, 1902. On the previous day I had seen him and made a diagnosis of general peritonitis following appendicitis of six days' duration. 'Two days later (Monday) his general condition was good, there was a tumour in the hypogastrium like a distended bladder, per rectum there were signs of encapsulated fluid in the rectoresical pouch which was bulging down the anterior rectal wall. The same day the abscess was opened per rectum. Four hours later the boy collapsed, vomited bloody fluid, and died late on night of operation. Necropsy : General and died late on night of operation. Necropsy : General coils of small intestine. The remains of a large abscess were found in the pelvis. There was a large collection of pus in the hepatic pouch and extending up over the liver. The appendix was long and hanging down into the pelvis, its end was gangrenous, and it contained an enterolith. Pus collections between intestinal coils were large and appeared to be residual. Note on Monday (day before death) the patient seemed so well that my previous diagnosis of general septic peritonitis was gravely doubted. On Tuesday (day of operation) the boy appeared to be in excellent condition, and at the operation it was remarked how safe and easy a proceeding operation it was remarked how safe and easy a proceeding
the opening of a pelvic abscess per rectum was. His death was unexpected and the post-mortem revelation a surprise."

\section{Rectal Tenesmus.}

Rectal tenesmus in any case should demand a rectal examination. In the ordinary pelvic abscess case it is likely to be an urgent and typical symptom. It is due to the abscess pressing upon the anterior wall of the rectum and causing œedema and bulging of the wall. Associated with it later is a spurious mucous diarrhoea and frequently sphincter paresis" (p. 512).

In women the diagnosis is more difficult than in men, and I have many notes of cases of pelvic peri- tonitis due to other acute conditions mistaken for appendicitis-such as ovarian cysts with twisted pedicles, leaking ectopic gestations, gonorrhoeal salpingitis, pyelitis, and stones in the lower end of the ureter. All of these may require a careful history and skilled examination for their differential diagnosis. The following notes will serve as an example of these manifold difficulties :-

CAsE 5 (p. 381).-Pelvic appendicitis in a female; pus draining through the Fallopian tube. A single woman, age 27 years, was admitted to the Royal Infirmary, Newcastle, on Sept. 26th, 1899 . On the 21st, while in bed, she felt a sudden pain about the stomach, and vomited. On the 22 nd she took some castor oil which was vomited. An enema administered later brought away some freal matter. On the $23 \mathrm{rd}$ the pain settled in the right iliac region, and she continued to vomit. On the 24 th and 25 th she had difficulty in passing urine, but her bowels were moved. The pain and vomiting continued. On admission to the infirmary at 9 A.M. she was flushed, had a coated tongue, and a temperature of $100 \cdot 8^{\circ} \mathrm{F}$., and a pulse of 120 . The whole abdomen was slightly distended, tender, and rigid. The note over the lower abdomen was dull on light percussion. Per rectum nothing was found. The patient was menstruating and passed through a normal period. After her admission the pain abated, the vomiting ceased, and four days later her temperature became and remained normal. On Oct. 5th. ten days after her admission, as a pelvic swelling was still palpable from the abdomen, she was examined under an anæsthetic. An indefinite firm mass was felt in the left iliac region. Per vaginam the hymen was found to be intact and the vagina virginal. A soft, fluid, immovable swelling, apparently of the size of a foetal head, bulged into and filled the anterior and lateral vaginal fornices. 'The bladder was emptied by a catheter and the swelling found to be entirely in front of the uterus. This was confirmed by the uterine probe. On withdrawing the uterine probe some fotid pus escaped from the cervical canal. The diagnosis suggested was that of suppurating dermoid ovarian cyst ruptured into a Fallopian tube. Operation was performed on Oct. 10 th. The abdomen was opened from the umbilicus to the pubes, -when the pelvis was seen to be filled by a mass covered by adherent intestine. The abdominal cavity was packed with sterile gauze, and the patient was placed in the Trendelenberg posture. On separation of the intestinal coils a large quantity of very offensive pus escaped and was quickly mopped up. The pouch of Douglas was practically a large abscess cavity, and the abscess extended upwards into the left iliac fossa. A fæcal concretion was found lying at the bottom of the pelvis, and on drawing the adherent cæcum forward a sloughy opening of the size of a sixpenny piece was found in it at the position occupied by the insertion of the appendix. The whole appendix had sloughed off. The right side of the abscess had been drained by the right Fallopian tube, which was slightly thickened but pervious, and had stinking pus in its lumien. The opening in the cæcum was sutured, the right Fallopian tube excised, and a large drain opening made from the bottom of Douglas's pouch into the vagina; the abdominal wound was entirely closed except at the lower end, where a glass tube was left. Cultivations from the gauze drain removed on Oct. 14th, four days after the operation, showed the Bacillus coli communis only. On the 18th, eight days after the operation, there was a free discharge of fæcal matter and pus from the vagina, and the temperature rose to $102^{\circ}$ This discharge continued, and on the 24 th she was very $i l l$, with a profuse discharge of fæces and pus from the vagina and from the lower part of the abdominal wound, and a temperature of $103^{\circ}$ in spite of frequent douching through a tube introduced into the vaginal opening. Constant irrigation was now adopted, and the cavity was flushed through from the vagina to the abdominal wound with sterile hot saline solution day and night. If the irrigator was stopped for a few hours her temperature rose and the irrigator had to be restarted. It was continued until Nov. 9th. She was discharged nearly healed on the 20th. In September, 1900, she returned to have a small ventral hernia, of the size of the drainage-tube, at the lower part of the ventral scar, operated upon. She was in good health, and bimanual examination could discover no sign of adhesions or any other abnormality in the pelvis.

\section{Treatment.}

The chief aim of this, in pelvic as in all cases of appendicitis, is to make a sufficiently early diagnosis to allow of removal of the appendix before it has ruptured, infected the peritoneum and caused an abscess. No one will now dispute this, though it is still too often a counsel of perfection, but considering the progress already made, is one capable of achievement if sufficient care and thought he brought to bear on each case. Within the first two days of the 
illness I think all will agree that the appendix should be removed through an abdominal incision, and if the escaping pus is offensive or in large quantity, that the pelvis should be drained, preferably by a glass tube kept dry by frequent emptying with a syringe and long rubber tube attached (p. 884). If this be done I have drawn attention to the need of protecting (or if this is not possible of dividins and ligatuning) the deep epigastric artery to prevent secondary hæmorrhage from it (p. 822). When definite signs of a pelvic abscess are discovered after the fourth day, especially if the small intestines are distended, and the patient is still ill-looking, I believe that an immediate operation does not give the patient his best chance, and may be fatal. Experience has impressed upon me the importance of waiting, watching, and medical treatment (p. 536). Some pelvic collections find their way into the adjoining intestine never so far as I know into the general peritoneal cavity, and recovery follows; more of them after a time increase in size and require operation, but this must be postponed till rectal tenesmus and bulging of the rectal wall make relief necessary.

I first advocated drainage through the rectum in $1896^{2}$ and $\mathrm{my}$ faith in the operation has steadily increased, because it is one of the safest and most satisfactory in surgery if properly performed at a suitable time. In women the abscess may often be, perhaps more conveniently, opened into the posterior vaginal fornix. Very ill and suffering patients are restored almost at once, and in three or four days have quite recovered their appetite and their feeling of well-being. If premature attempts are made to open the abscess, it becomes difficult to keep it drained, and repeated and dangerous explorations may have to be done. If, on the other hand, courage and patience are exercised until the abscess is "ripe," a strip of iodoform gauze left in the opening and projecting from the anus suffices to keep the wound open long enough to complete the relief. Even this I seldom touch, but allow it to come away by itself with the first bowel movement. No further plugging or drainage are necessary or useful.

My belief, based upon considerable post-mortem and clinical experience. is that the boy (Case 4) would have recovered possibly without, but more probably with, surgical aid limited to drainage of abscesses if operation had been postponed. Any time after a fortnight of complete recovery the appendix should be excised.

\section{Normal Resistance of the Peritoneum and Acquired} Peritoneal Immlunity.

Twenty-eight vears ago (p. 72), in a paper contributed to the Edinburgh Medical Journal at a time when the advance of abdomina! surger $r$ was being hindered by the old dread of opening the peritoneum, I approached this question as follows:-

On several occasions I have observed in abdominal wounds dressed for the first time on the tenth day, that though the superficial parts were suppurating, the peritoneum was not involved-both, of course, having been exposed to the same deleterious influences. I have also noticed, not without surprise, that rougher surgery may be perpetrated with impunity inside of the peritoneum than would be tolerated by any portion of the organism outside of it. The unavoidable conclusion is that the peritoneum has a resisting power not belonging to other tissues."

It seems now, at a time when one of the main objects of operation is to prevent peritonitis. almost impossible to realise that any such dread existed about inflammation of the peritoneum. I have already conveyed a hint in connexion with immunity, that for tubular organs this is largely acquired, and I propose to suggest a principle bearing upon this, applicable to every part of the body. During the last week an intelligent patient came under ny care with a synovitis following "locking" of his knee-joint. He had had two operations for the removal of a loose cartilage previously, both of which had failed to remove his disability. A large incision into the joint disclosed a rim of loose

Brit. Mred. Jonu, 1896, ii., 1005. internal cartilage left attached round the inner side of his joint and a lipomatous mass. Both were excised. 'The first operation had been followed by much pain, the second by less, and the third caused scarcely any disturbance at all. This is not an exceptional experience, but is the rule in similar cases.

\section{Acquired Peritoneal Immunity.}

The older surgeons recognised that ovariotomy on a patient who had been tapped and had peritonitis, although perhaps requiring a much more difficult and tedious operation, was followed by less disturb ance and smaller mortality than were simple cases without adhesions. They offered no explanation of this experience, but acknowledged it as a curious phenomenon which seenied to be inexplicable. It is not. Acquired immunity of the peritoneum is an asset of great value to the surgeon, and one that has not yet received due recognition. A first operation in the abdomen is followed by what $I$ have called a Trinity of Terrors. The patient is less or more sickly for 24, thirsty for 48 , and griped for 72 hours. After a second operation these symptoms, if they appear at all, are scarcely disturbing, and after recovery from an acute infection the peritoneal cavity has acquired such a degree of inmunity that it is scarcely possible to infect it again. Every observant surgeon must have noted the safety of gastrectomy or colectomy done in two stages. It is unfortunate that a double-barrelled operation promotes the dissemination and recurrence of cancerous growths in the abdomen, or this would become the favoured method of dealing with these cases.

As illustrating the importance of acquired immunity the following record will suffice.

CASE 6 (p. 607).- " The patient, a female, aged 35, was operated upon first on June 10th, 1902. She was then very ill, with an acute abdominal illness diagnosed as abscess in the pelvis and in connexion with the vermiform appendix. Her abdomen was opened over the right iliac fossa by an incision extending from the loin behind. Some clear fluid escaped on opening the peritoneum. The colon and the cæcum were adherent to the parietal peritoneum on the outer side, and on gently separating them foetid pus escaped. The appendix was found lying on the outer side of the cæcum and colon, with its tip pointing upwards to the hepatic pouch, and was excised. The pelvic brim was filled with matted adherent intestine. The abdominal wound was covered up, and the patient was placed in the lithotomy posture, the pelvic abscess, from which a large amountabout one pint-of foetid pus was evacuated, being opened and drained through an incision in the posterior vaginal fornix by drainage-tubes sewed in. The vermiform appendix contained a stercolith, and was perforated at its centre."

In a month the patient had recovered and had arranged to go home (July 14th, 1902), when she was suddenly seized with symptoms of acute intestinal obstruction and became very ill.

"The second operation was performed a few hours later The abdomen was opened in the middle line. The smal intestines were congested and beginning to distend, a distended loop leading to the pelvis where the obstruction wa located. It was due to adhesions, many of which were easily separated, but those in the depths of the pelvis became more dense. In separating a loop there a small abscess wa opened and pus escaped. Traction on the inflamed and adherent loop resulted in tearing the intestine almost completely across. Six inches of torn and adherent intestine were excised, and an end-to-end anastomosis was made by catgut sutures. 'The pelvic abscess was thoroughly mopped. and the whole pelvis was washed out with saline solution. The abdomen was entirely closed and the patient recovered."

Intestinal Obstruction as a Result of Appendix Abscess.

In this case obstruction symptoms were delayed for one month ; in the majority of cases they commence during the second or third week after drainage of the abscess. Since the use of gauze for drainage purposes was discontinued, obstruction has been a rare event in ordinary abscess cases, but it still follows pelvic abscess with disconcerting frequency. Fortunately, recovery has advanced so far that recognition of the complication is easy, and if an early diagnosis is promptly followed by operation recovery can be counted upon, in spite of the presence of an active infection. Recurrence of obstruction must be rare, 
for I have never seen a case, but patients' friends should be warned of possibilities, as late obstruction can occur long after the attack has been recovered from; I have notes of cases as long as ten years afterwards. The following example will suffice to illustrate these points :-

CASE 7 (p. 621).- " Female, aged 40, admitted to Royal Victoria Infirmary, Newcastle, on Dec. 30th, 1902. Sho had been operated upon in March, 1900, for appendicitis with abscess. A pelvic abscess which developed during convalescence was opened from the vagina. Until two weeks ago she had been quite well. Two weeks ago she hacl a sudden attack of pain in the stomach. It was a continuous pain and made her vomit. Her bowels had not been moved pince. On Dec. 31 st, after a dose of castor oil (1 oz.) her since. On Dec. 31st, after a dose of castor oil (1 oz.) her but she still complained of pain in the left hypochondrium. Jan. 1st, 1903, she was fairly well till the afternoon, when acute pain commenced and she began to vomit. At midnight there was moderate distension and visible peristalsis. The vomited matter was only food she had taken. After a simple enema her bowcls were well moved.

Jan. 2nd, 1903, I found as follows : The patient looked ill and complained greatly of griping intermittent pains. Her hands and face were cold, pulse 128 and very soft, tongue dry in the centre. There was a median scar on the abdominal wall between the pubis and the umbilicus. The lower part of the abdomen was slightly distended. Intestinal movements were so vigorous as to be obvious. The right hepatic region was tender. No mass was felt. P.V., uterus pushed back and fixed.

Operation (Jan. 2nd).-Immediately after the conclusion of the examination $\frac{1}{3}$ gr. of morphia was injected hypodermically. Local anæsthesia was then induced by infiltration with eucaine. A few drops (not more than 25 min.) of chloroform were admintstered by inhalation as a placebo. Enterostomy was done in the right flank by opening the first presenting and distended loop of small intestine. A L'aul's tube was tied into the bowel. The operation took a quarter of an hour. The operation was well borne and there was very little pain. The tube discharged brown fæcal matter, but though there was relief of all urgent symptoms she never picked up, and died five days later.

Necropsy. - There was no gross evidence of general septic peritonitis, but the intestines were sticky and slightly adherent. The enterostomy opening was found to be at one-third of the distance from the duodenum to the ileo-cæcal valve. The small intestine, both above and below the enterostomy opening, as far indeed as the seat of obstruction, was distended with gas. The obstruction was in the pelvis. A loop, 6 inches long, of small intestine was strangulated under a band (the result of old peritonitis). The band passed from the cæcum to the mesentery of the loop of intestine which had slipped under it. The constriction was not very tight, and there was no gangrene either of the strangulated loop or of the part over which the band passed. The obstruction was at the commencement of the lower one-third of the small intestine; and below this the bowel was collapsed, and the coils adherent to one another, to the pelvic wall and to the pelvic viscera. There was no disease of any other organ. As evidence of an old general peritonitis, there were, in addition to the pelvic adhesions, adhesions between both lobes of the liver and the diaphragmatic peritoneum. No cause for this but the previous attack of appendicitis was found."

Another problem raised by Case 6 is the manner of dealing with the adherent intestine to avoid tearing of it in such difficult cases-and I think I can offer a solution based upon subsequent experiences. Instead of endeavouring to separate the coils by traction it is best to introduce a hand and find the line of cleavage between the bladder and clumped intestine in the male, and the uterus and the intestine in the female. With the back of the hand towards the bladder or uterus, and the palm facing the intestines and closely hugoing the bladder or uterus, the fingers are insinuated between them to the pelvic floor, then keeping them curved, separation of adhesions below is continued in close contact with the recto-vesical pouch, when the wh le' of the adherent intestine, often wallowing in pus, can be drawn forward and out of the abdomen, where separation and if necessary repair can be more easily accomplished. If this operation can be performed early the patients are immediately relieved and seldom fail to recover, as peritonitis has ceased to be a serious danger because of their acquired immunity.

In six very serious and difficult cases, four of $\mathrm{my}$ own, one of Mr. W. G. Richardson, and one of Prof.
Brunton Angus, an operation suggested by me was carried out in each. All were women. In three of them a pelvic abscess due to appendicitis was the cause of the trouble, in three pelvic abscess from salpingitis. All had been operated upon, and after operation developed a frecal fistula through the wound. They wele all, with the exception of one case, very ill, with high temperature and free purulent and frecal discharge. A variety of treatments had failed to help them and death seemed to be inevitable unless something more could be done. In all six vaginal hysterectomy was performed, the broad ligaments were secured in clamp forceps, and the pelvis between the forceps and surrounding them was packed with strands of iodoform gauze to allow of free pelvic drainage. All recovered from the operation, and the five acute cases made a complete recovery within three months after the operation. The less ill case (pyosalpinx) improved, but a year later was reported to me as still having a freal fistula. Such an operation can seldom be necessary, because fæcal fistulre when buried (i.e., if the mucous membrane is not attached to the surface) seldom fail to close themselves. A record of this method may, however, be useful if an otherwise hopeless situation has to be faced, because the successes obtained now justify the proceeding.

\section{Conclusions.}

Inflammation of the pelvic appendix can still mislead diagnostic experts. The ordinary symptoms of appendicitis-abdominal pain and fever-may both be absent in pelvic appendix cases.

The severe pain often attending appendicitis in the early stages, wherever the organ is situated, is due to tension within the appendix resulting from retention of its contents. During this period the symptoms are similar to those of acute intestinal obstruction.

Prolonged tension results in gangrene, when acute pain disappears until perforation occurs and a second variety of pain due to peritoneal irritation suddenly derelops. In many pelvic appendix cases there is no severe early pain. Not until the appendix has perforated, generally at its distal end, are urgent symptoms noted. Absence of pain is not special to the pelvic appendix, but is more common in this than any other variety. The explanation offered is that tension is not present, because this type of appendis can empty itself, and the first serious pain results from perforation.

Frequency of micturition, especially in boys, and in rare cases retention of urine, occurs as an early symptom.

Careful rectal examination may save the situation in a difficult case. So may a blood count.

Pelvic abscess may result from the localisation of a diffuse peritoneal infection, from leaking of an abscess connected with the appendix in the right iliac fossa, or from a leaking pelvic appendix.

Rectal tenesmus is the most suggestive symptom of a pelvic abscess.

The treatment of an inflamed pelvic appendix is to remove it as soon as a diagnosis has been made. After the second day from perforation it is seldorn wise to operate from the abdomen, and this should never be done if the intestines are distended or the patient is acutely ill. Medical treatment is then the method of choice, until the abscess points in the rectum so prominently that it can be safely and easily opened there.

The normal peritoneum possesses an extraordinary natural immunity, but the importance of its acquired immunity is not yet appreciated fully.

Intestinal obstruction follows operations for pelvic appendicitis with disturbing frequency. When convalescence seems assured, during the second or third week, it develops suddenly. Early operation will save nearly every patient-delay is deadly. Following abdominal operation for pelvic abscess a complicated freal fistula may refuse to heal and threaten life. In women vaginal hysterectomy will save the majority of these dangerous cases. 